\title{
Hypersingular integral equations for a thermoelastic problem of multiple planar cracks in an anisotropic medium
}

\author{
W.T. Ang ${ }^{\mathrm{a}, *}$, D.L. Clements ${ }^{\mathrm{b}}$ \\ ${ }^{a}$ Computational and Mathematical Sciences Group, Faculty of Information Technology, Universiti Malaysia Sarawak, \\ 94300 Kota Samarahan, Malaysia \\ ${ }^{\mathrm{b}}$ Department of Applied Mathematics, University of Adelaide, Adelaide, SA 5001, Australia
}

Received 3 December 1998; accepted 12 August 1999

\begin{abstract}
The problem of calculating the thermoelastic stress around an arbitrary number of arbitrarily located planar cracks in an infinite anisotropic medium is considered. The cracks open up under the action of suitably prescribed heat flux and traction. With the aid of suitable integral solutions, we reduce the problem to solving a system of Hadamard finite-part (hypersingular) integral equations. The hypersingular integral equations are solved for specific cases of the problem. (C) 1999 Elsevier Science Ltd. All rights reserved.
\end{abstract}

Keywords: Thermoelastic stress; Multiple cracks; Hypersingular integral formulation; Anisotropic medium

\section{Introduction}

Anisotropic structures can now be found in an increasingly wider range of applications in modern technology. For example, synthetic materials, such as plywoods and carbon-carbon composites, which exhibit anisotropic behaviours are widely used in the design and construction of modern vehicles (e.g. aircrafts and high-speed trains). The need to assess the reliability and integrity of these structures has given rise to a vast body of literature on cracks in anisotropic media. The majority of the papers in the literature, e.g. Sollero and Aliabadi [1], Ang [2], Sweeney [3] and Stroh [4], ignored the effect of heat flow on the stress distribution around the cracks.

There are relatively fewer studies which analyse the thermoelastic stress around cracks in anisotropic solids. Using integral transform techniques, Atkinson and Clements [5] calculated the thermoelastic stress around a planar crack in an infinite anisotropic medium. Similar problems involving a single crack in an infinite anisotropic material were also solved by Hwu [6], Tsai [7] and Wu [8]. Sturla and Barber [9] extended the work of Atkinson and Clements [5] to allow for the possibility that the crack may be partially closed. Clements [10] examined the thermoelastic problem of a planar crack between bonded dissimilar anisotropic materials.

\footnotetext{
* Corresponding author. Tel.: +60-82-671000; fax: +60-82-672301.

E-mail address: wtang@mailhost.unimas.my (W.T. Ang)
}

In the present paper, the problem of calculating the thermoelastic stress around an arbitrary number of arbitrarily located planar cracks in an infinite anisotropic medium is considered. The problem is reduced to the task of solving a system of Hadamard finite-part (hypersingular) integral equations. The unknown functions in the integral equations are the jumps in the temperature across opposite crack faces and the crack-opening displacements. For convenience, we assume that the cracks open up under the action of suitable prescribed heat flux and traction. It may be worth mentioning that the analysis presented is valid for the most general anisotropic material, i.e. it does not require the material to possess any particular symmetries in its anisotropy.

\section{Temperature around cracks}

\subsection{Mathematical formulation}

By referring to a Cartesian coordinate system $0 x_{1} x_{2} x_{3}$, consider an infinite anisotropic elastic medium in which there are $N$ arbitrarily located planar cracks having geometries that do not vary along the $x_{3}$-axis. The cracks denoted by $C^{(1)}, C^{(2)}, \ldots$ and $C^{(N)}$ are assumed not to intersect with one another. On the $0 x_{1} x_{2}$ plane, the tips of a typical crack $C^{(k)}$ are given by $\left(a^{(k)}, b^{(k)}\right)$ and $\left(c^{(k)}, d^{(k)}\right)$. Refer to Fig. 1 .

The cracks are acted upon by suitably prescribed heat flux. The heat flux generated by the cracks is required to 